\section{Novel dim target detection and estimation algo- rithm based on double threshold partial differential equation}

\author{
Meng Li, T. X. Zhang, Z. R. Zuo, X. C. Sun, and \\ W. D. Yang \\ Huazhong University of Science and Technology, State \\ Education Commission Laboratory for Image Processing \\ and Intelligent Control, Institute for Pattern Recognition \\ and Artificial Intelligence, Wuhan 430074, China
}

\begin{abstract}
The aim of the present work is to propose a brand-new algorithm based on an adaptive double threshold nonlinear anisotropic diffusion equation (DTPDE) to detect and track moving dim targets against complex cluttered background in infrared (IR) image sequences. We also illustrate the performance comparisons of the proposed algorithm DTPDE and two-dimensional least mean squares (TDLMS) on real IR image sequence data. Extensive experiment results demonstrate the proposed novel algorithm's flexibility and adaptability in detecting moving weak dim targets. () 2006 Society of Photo-Optical Instrumentation Engineers. [DOI: 10.1117/1.2354504]
\end{abstract}

Subject terms: weak dim target detection; double threshold partial differential equation; two-dimensional least mean squres; infrared image.

Paper 050950LRR received Dec. 9, 2005; revised manuscript received Jun. 24, 2006; accepted for publication Jun. 28, 2006; published online Sep. 19, 2006.

\section{Introduction and Motivation}

A crucial problem in spaceborne and IR surveillance systems today is the detection and recognition of weak moving targets at low signal-to-noise/clutter ratios. Algorithms are currently available to reduce background clutter and random noise and to enhance target detectability. Early work in infrared search and track systems was adequate for early applications in which the targets were bright compared to the background clutter. ${ }^{1,2}$ For situations in which the noise in the entire scene does not follow a particular model, partitioning the images into areas with different noise characteristics was proposed. ${ }^{3}$ Tarun, Zeidler, and $\mathrm{Ku}^{4}{ }^{4}$ and Lin, $\mathrm{Nie}$, and Unbehauen ${ }^{5}$ studied the performance of a spatial filter based on two-dimensional least mean square (TDLMS) adaptive filters and local-mean removal filters for the detection of small objects in image data.

The algorithms mentioned are isotropic high-pass filters commonly used in the field of dim target detection, but it is difficult for them to smooth edges caused by image texture. We are eager to discover a useful way to overcome this major disadvantage. To solve this problem, the challenge is to design methods that can selectively smooth a noisy image without losing significant features. A partial differential equation (PDE) is the best choice. Since it was introduced by Perona and Malik, ${ }^{6}$ much research has been devoted to

0091-3286/2006/\$22.00 @ 2006 SPIE its theoretical and practical understanding. Black et al. ${ }^{7}$ studied the relation between PDE and robust statistics. You and Kaveh, ${ }^{8}$ and Lysaker, Lundervold, and $\mathrm{Tai}^{9}{ }^{9}$ used fourth-order PDE to optimize the trade-off between noise removal and edge preservation, and to overcome the staircase effect that occurs with the TV-norm filter. Scharr and Spies ${ }^{10}$ combined 3-D anisotropic diffusion and motion estimation for denoising and improvement of motion estimation.

Our primary motivation was to propose a novel algorithm to further smooth the edge texture and promote the ability of detecting weaker targets. In Sec. 2 we introduces the Perona and Malik PDE model. In Sec. 3 we develop a novel background clutter and random sensor noise suppression method based on double threshold PDE (DTPDE). In Sec. 4 we present the application of a new algorithm and analysis the comparison of the new algorithm and TDLMS target detection algorithm. We come to a conclusion in Sec. 5 .

\section{Perona and Malik Anisotropic Diffusion Model}

Perona and Malik proposed a nonlinear diffusion method for avoiding the blurring and localization problems of linear diffusion filtering. They applied an inhomogeneous process that reduces the diffusivity at those locations, which have a larger likelihood to be edges. This likelihood is measured by $c(\|\nabla I\|)$.

$\partial I(x, y, l) / \partial t=\operatorname{div}[c(\|\nabla I\|) \nabla I]$,

where $I(x, y, l)$ is the diffused image. $l$ determines the diffusion time. $\nabla I$ is the image gradient, and $c(\|\nabla I\|)$ is a non-negative diffusion coefficient threshold function formulated as:

$$
\begin{aligned}
c(\|\nabla I\|)= & 1 /\left(1+\|\nabla I\|^{2} / K^{2}\right) K>0 \text { or } \\
& c(\|\nabla I\|)=\exp \left\{-(\|\nabla I\| / K)^{2}\right\} K>0 .
\end{aligned}
$$

Equation (1) can be discretized as follows, using a fournearest-neighbors discretization of the Laplacian operator:

$I_{s}^{l+1}=I_{s}^{l}+\lambda \sum_{p \in \eta_{s}} g\left(\nabla I_{s, p}\right) \nabla I_{s, p}$,

where $I_{s}^{l}$ is the discretely sampled image, $s$ denotes the pixel position, and $l$ is the discrete time step iterations. The constant $\lambda \in \mathbb{R}^{+}$is a scalar that determines the diffusion rate, and $\eta_{s}$ represents the spatial neighborhood of pixel $s$. Perona and Malik linearly approximated the gradient in a particular direction as

$\nabla I_{s, p}=I_{p}-I_{s}^{l}$

\section{Proposed Target Detection Algorithm- Double-Threshold Partial Differential Algorithm}

Detection of targets against complex cluttered background is generally impossible using only a single frame. The image sequence contains randomly drifting cluttered background and may also be contaminated by random sensor noise. Since the field of view is relatively fixed with respect to the background, the complicated background clutter can be removed by a prewhitening method. The usual way is 


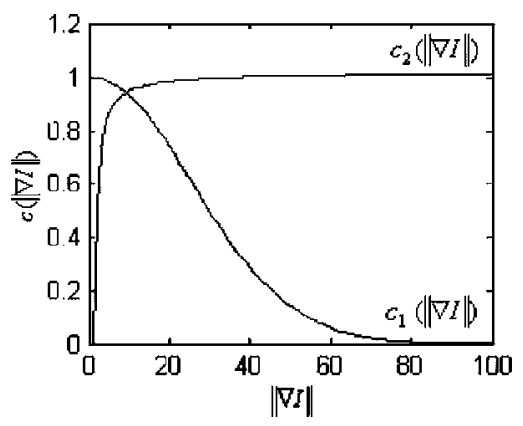

Fig. 1 The performance of $c_{1}(\|\nabla /\|)$ and $c_{2}(\|\nabla /\|)$.

local mean removal, two-dimensional least mean square (TDLMS) adaptive filters, or differencing operation, etc. But they are useless for the texture change of background, since they are isotropic filters. If the target velocity is small, the targets will be eliminated by the differencing way. And those methods are all sensitive to the image shifting. Here, we propose a novel algorithm based on DTPDE to overcome those shortcomings. The proposed algorithm works quite well for detecting moving targets against complex cluttered background and random noise. It is composed of four parts: the DTPDE background estimation algorithm, clutter eliminating, random noise suppression, and target trajectories estimation.

We mainly present the first part-the DTPDE background estimation algorithm. In contrast with isotropic filters like TDLMS, the PDE process is twofold: the edges remain stable while noise is smoothed locally. The main mission of target detection in the complex cluttered background is to suppress the complicated clutter. The proposed algorithm DTPDE improves on the PDE algorithm to satisfy the practical application of executing the task of recovering a good estimate of the true image from a degraded observation. It can be formulated as follows:

$$
\begin{aligned}
I_{s}^{l+1}= & I_{s}^{l}+\lambda \sum_{p_{1} \in \eta_{s 1}, p_{2} \in \eta_{s 2}} c_{1}\left(\nabla I_{s, p_{1}}\right)\left\{c_{2}\left(\nabla I_{s, p_{2}}\right) \nabla I_{s, p_{1}}\right. \\
& \left.+\left[1-c_{2}\left(\nabla I_{s, p_{2}}\right)\right] \nabla I_{s, p 2}\right\}
\end{aligned}
$$

where $\eta_{s 1}$ and $\eta_{s 2}$ represent the eight-nearest and fournearest spatial discretization neighborhood of pixel $s$, respectively. $c_{1}(\|\nabla I\|)$ is a diffusive coefficient threshold function as described in Eq. (4), $c_{2}(\|\nabla I\|)$ is the second diffusive coefficient threshold function formula as

$$
\begin{aligned}
c_{2}(\|\nabla I\|)= & K_{2}\left[\exp \left(\|\nabla I\|^{2}\right)-\exp \left(-\|\nabla I\|^{2}\right)\right] /\left[\exp \left(\|\nabla I\|^{2}\right)\right. \\
& \left.+\exp \left(-\|\nabla I\|^{2}\right)\right] .
\end{aligned}
$$

$\nabla I_{s, p_{1}}$ and $\nabla I_{s, p_{2}}$ are formulated as

$\nabla I_{s, p_{1}}=I_{p_{1}}-I_{s}^{l}$ and $\nabla I_{s, p_{2}}=I_{p_{2}}-I_{s}^{l}$.

Figure 1 depicts the performance of the two "edgestopping" threshold functions $c_{1}(\|\nabla I\|)$ and $c_{2}(\|\nabla I\|)$. As we will see, the choice of $c_{1}(\|\nabla I\|)$ and $c_{2}(\|\nabla I\|)$ can greatly affect the extent to which discontinuities are preserved. Understanding this is one of the main goals of this work.

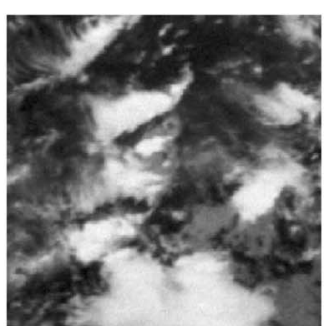

(a)

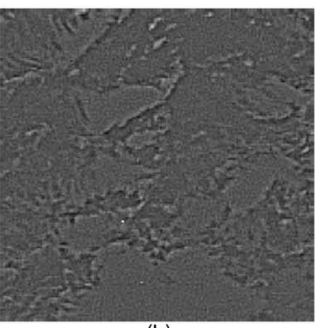

(b)

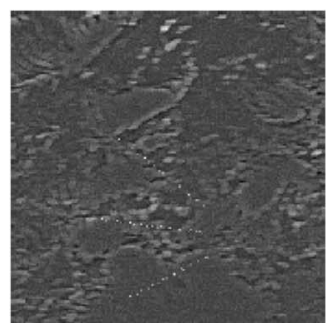

(d)

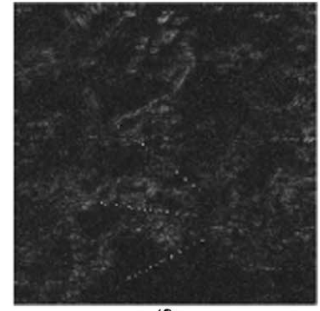

(f)

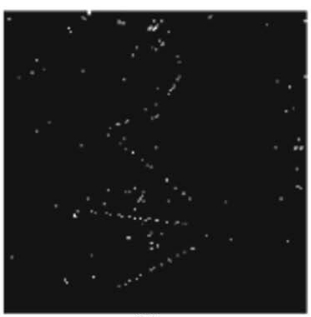

(h)

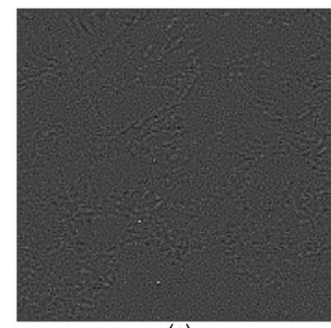

(c)

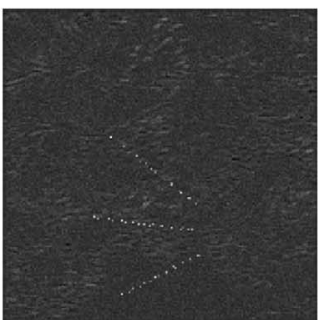

(e)

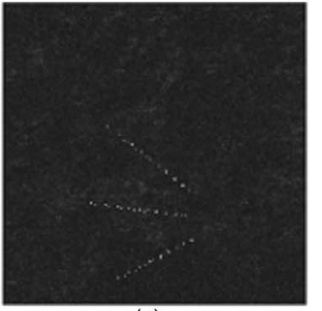

(g)

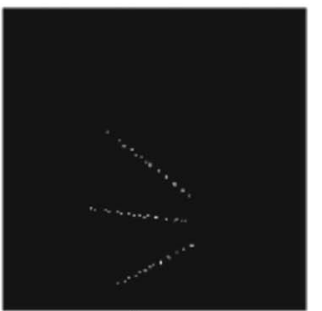

(i)
Fig. 2 Performance comparison of TDLMS and the proposed algorithm. (a) First frame of the initial image sequence $l(x, y, t)$, (b) first frame of the TDLMS's results, (c) first frame of DTPDE's results, (d) TDLMS's results projecting on $t$ coordinate, (e) DTPDE's results projecting on $t$ coordinate, (f) time-predicting results of TDLMS's results projecting on $t$ coordinate, (g) time-predicting results of DTPDE's results projecting on $t$ coordinate, (h) segment result of (f), and (i) segment result of $(\mathrm{g})$.

It is obvious that the texture edges remain sharp and stable, while smoothing takes place only inside the regions and acts even more effectively. This can be mathematically explained by analysis of the threshold function $c_{1}(\|\nabla I\|)$ and $c_{2}(\|\nabla I\|)$. In $c_{1}(\|\nabla I\|)$, for large values of $K$, we have small participation of the gradient and thus a large diffusion effect; inversely, for small values of $K$, we have much par- 
Table $1 \mathrm{ISCR}_{/}$and $\mathrm{ISCR}_{g}$ of different algorithms.

\begin{tabular}{lccccc}
\hline \hline & Original image & TDLMS & DTPDE & TDLMS+TPA & DTPDE+TPA \\
\hline $\mathrm{ISCR}_{l}$ & 1.4798 & 3.2325 & 6.3573 & 4.4328 & 6.8236 \\
$\mathrm{ISCR}_{g}$ & 0.2672 & 5.3341 & 7.8712 & 6.2145 & 8.2872 \\
\hline \hline
\end{tabular}

ticipation of the gradient and small diffusion. And if we have another look at the function $c_{2}(\|\nabla I\|)$, it is just the contrary. The DTPDE method uses two thresholds to detect strong and weak edges separately. In the smooth area with small gradient, we use isotropic diffusion to eliminate noises and dim targets. In the edge area with great gradient within the two thresholds, we use double threshold anisotropic diffusion to eliminate noises and dim targets, but keep the edges sharp and stable. Outside of the two thresholds, we ignore it.

The constants $K$ and $K_{2}$ were decided by using the noise estimator described by Canny; a histogram of absolute values of the image gradient was computed. $K$ and $K_{2}$ were set equal to $c_{1} \%$ and $c_{2} \%$ value of their integral at every iteration, respectively, and were decided by the image and real application requirements.

After using the DTPDE algorithm to estimate the cluttered background, we use subtraction to eliminate the cluttered background from the initial image $I(x, y, t)$. It can be formulated as

$I_{\vec{s}}(x, y, t)=I(x, y, t)-I_{s}^{l+1}(x, y, t)$.

Then, we can use a multiframe accumulation method, or autocorrelation or time-predicting algorithm, or velocity filtering theory to suppress the random noise. Considering the trade-off between performance and computer complexity, we chose a time-predicting algorithm (TPA). It can be expressed in the following formula:

$\widetilde{I}_{\widehat{s}}(x, y, t)=\omega I_{\widehat{s}}(x, y, t)+(1-\omega) \widetilde{I}_{\widehat{s}}(x, y, t-1)$.

After the cluttered background and random noise suppression, we can use dynamic programming, pipeline filtering, the Hough transform, or track matching algorithm to estimate the target trajectories.

\section{Experiments and Results}

After cluttered background and random noise suppression, the initial image sequence $I(x, y, t)$ is transformed into $\tilde{I}_{\widehat{s}}(x, y, t)$, which has whitening structures and characteristics. To evaluate the background clutter and random noise suppression ability, we define the local integrated signal to clutter ratio $\left(\mathrm{ISCR}_{l}\right)$ and global integrated signal to clutter ratio $\left(\mathrm{ISCR}_{g}\right)$ as follows,

$$
\begin{aligned}
\mathrm{ISCR}_{l}= & \mu_{s} / \sigma_{c l}=\left|\mu_{b s}-\mu_{b}\right| / \sigma_{c l} \text { and } \\
& \mathrm{ISCR}_{g}=\mu_{s} / \sigma_{c g}=\left|\mu_{b s}-\mu_{b}\right| / \sigma_{c g},
\end{aligned}
$$

where $\mu_{b s}$ is the intensity value of the target, and $\mu_{b}$ is the average intensity value of the pixels in the neighbor area around the target. $\mu_{s}$ is the relative value of the target. $\sigma_{c l}$ is the standard variance of the neighbor area around the target, and $\sigma_{c g}$ is the image's standard variance.

Figure 2 depicts the performance comparison of TDLMS and the proposed algorithm. The shifting magnitude of $I(x, y, t)$ is two pixels per frame. We list ISCR of $I(x, y, t)$, TDLMS, and the proposed methods in Table 1.

\section{Conclusion}

We propose a novel target detection algorithm based on DTPDE. It is heuristically demonstrated that the proposed algorithm is very successful for the task of detection and tracking of pixel-sized target trajectories. We illustrate the performance comparisons of the proposed method and TDLMS algorithm applied to IR images in real-world conditions. The experiment results prove that our improved algorithms promote the ability to detect weaker targets with seriously cluttered image sequences with very low signalto-noise ratio.

\section{Acknowledgments}

This work was supported by the National Natural Science Foundation of China (number 60135020).

\section{References}

1. D. S. K. Chan, D. A. Langan, and D. A. Stayer, "Spatial processing techniques for the detection of small targets in IR clutter," Proc. SPIE 1305, 53-62 (1990).

2. M. Fernandez, A. Aridgides, and D. Bray, "Detecting and tracking low-observable targets using IR," Proc. SPIE 1305, 193-206 (1990).

3. K. A. Melendez and J. W. Modestino, "Spatio-temporal multiscan adaptive matched filtering," Proc. SPIE 2561, 51-65 (1995).

4. S. Tarun, J. R. Zeidler, and W. H. Ku, "Performance evaluation of 2 -D adaptive prediction filters for detection of small objects in image data," IEEE Trans. Image Process. 2(3), 327-339 (Jul. 1993).

5. J. N. Lin, X. Nie, and R. Unbehauen, "Two-dimensional LMS adaptive filter incorporating a local-mean estimator for image processing," IEEE Trans. Circuits Syst., II: Analog Digital Signal Process. 40(7), 417-428 (Jul. 1993).

6. P. Perona and J. Malik, "Scale-space and edge detection using anisotropic diffusion," IEEE Trans. Pattern Anal. Mach. Intell. 12(7), 629639 (Jul. 1990).

7. M. J. Black, G. Sapiro, D. H. Marimont, and D. Heeger, "Robust anisotropic diffusion," IEEE Trans. Image Process. 7(3), 421-432 (1998).

8. Y. L. You and M. Kaveh, "Fourth-order partial differential equations for noise removal," IEEE Trans. Image Process. 12(12), 1723-1730 (Oct. 2000).

9. M. Lysaker, A. Lundervold, and X. C. Tai, "Noise removal using fourth-order partial differential equation with applications to medical magnetic resonance images in space and time," IEEE Trans. Image Process. 9(10), 1579-1590 (Dec. 2003).

10. H. Scharr and H. Spies, "Accurate optical flow in noisy image sequences using flow adapted anisotropic diffusion," Signal Process. Image Commun. 20, 537-553 (2005). 